
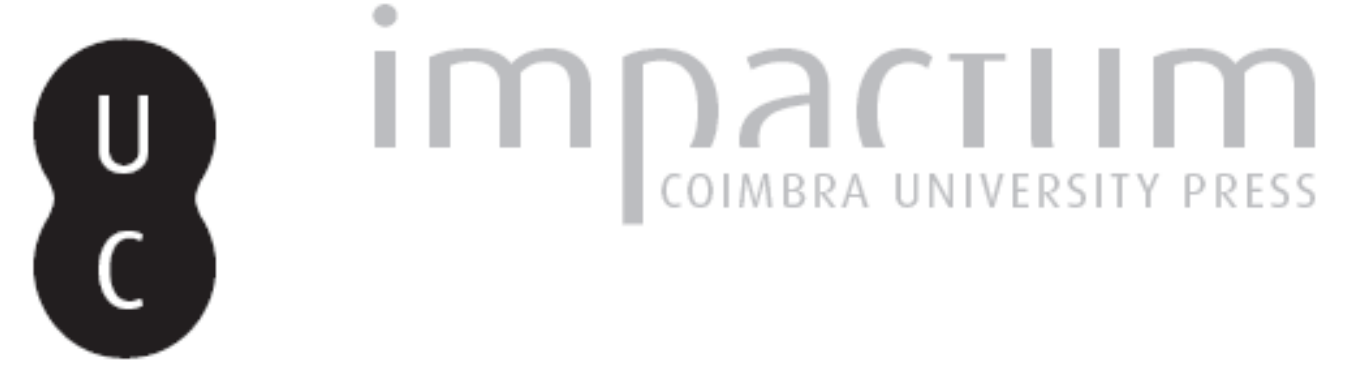

\title{
Dimensão espacial do(s) risco(s) em Portugal continental e na área metropolitana do Porto: perceção dos alunos do 9. ano de escolaridade
}

Autor(es): $\quad$ Martins, Bruno; Nunes, Adélia; Lourenço, Luciano

Publicado por: Imprensa da Universidade de Coimbra

URL

persistente:

URI:http://hdl.handle.net/10316.2/44196

DOI:

DOI:https://doi.org/10.14195/1647-7723_25-2_8

Accessed : $\quad$ 26-Apr-2023 11:11:55

A navegação consulta e descarregamento dos títulos inseridos nas Bibliotecas Digitais UC Digitalis, UC Pombalina e UC Impactum, pressupõem a aceitação plena e sem reservas dos Termos e Condições de Uso destas Bibliotecas Digitais, disponíveis em https://digitalis.uc.pt/pt-pt/termos.

Conforme exposto nos referidos Termos e Condições de Uso, o descarregamento de títulos de acesso restrito requer uma licença válida de autorização devendo o utilizador aceder ao(s) documento(s) a partir de um endereço de IP da instituição detentora da supramencionada licença.

Ao utilizador é apenas permitido o descarregamento para uso pessoal, pelo que o emprego do(s) título(s) descarregado(s) para outro fim, designadamente comercial, carece de autorização do respetivo autor ou editor da obra.

Na medida em que todas as obras da UC Digitalis se encontram protegidas pelo Código do Direito de Autor e Direitos Conexos e demais legislação aplicável, toda a cópia, parcial ou total, deste documento, nos casos em que é legalmente admitida, deverá conter ou fazer-se acompanhar por este aviso.

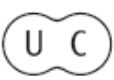




\title{
DIMENSÃO ESPACIAL DO(S) RISCO(S) EM PORTUGAL CONTINENTAL E NA ÁREA METROPOLITANA DO PORTO: PERCEÇÃO DOS ALUNOS DO 9. ANO DE ESCOLARIDADE*
}

\author{
SPATIAL DIMENSION OF THE RISK(S) IN PORTUGAL MAINLAND AND THE PORTO METROPOLITAN AREA: \\ $9^{\text {TH }}$ GRADE STUDENTS' PERCEPTION
}

Bruno Martins

Departamento de Geografia e Turismo, CEGOT, NICIF, Universidade de Coimbra (Portugal) ORCID 0000-0001-8681-2349 bruno.martins@uc.pt

Adélia Nunes

Departamento Geografia e Turismo, CEGOT e RISCOS, Universidade de Coimbra (Portugal) ORCID 0000-0001-8665-4459 adelia.nunes@fl.uc.pt

Luciano Lourenço

Departamento Geografia e Turismo, CEGOT e RISCOS, Universidade de Coimbra (Portugal) ORCID 0000-0002-2017-0854 luciano@uc.pt

\section{RESUMO}

Neste estudo pretende-se aferir a perceção que os alunos do $9^{\circ}$ ano de escolaridade têm sobre alguns conceitos fundamentais ligados à ciência do Risco e avaliar a respetiva capacidade em identificar e classificar os riscos naturais e mistos com maior probabilidade de ocorrência, tanto à escala nacional como na Área Metropolitana do Porto (AMP). Procedeu-se à aplicação de um inquérito, por questionário, e os resultados mostram que os discentes classificaram o risco de incêndio florestal como o de maior probabilidade de ocorrência, quer a nível nacional quer no conjunto dos concelhos da AMP, com uma classificação a variar entre elevada e moderada, respetivamente. A nível municipal, são de destacar algumas diferenças no "ranking" definido para as diferentes tipologias de risco. 0 estudo sugere, ainda, que a maioria dos alunos tem dificuldade em identificar a génese, as consequências e as medidas de mitigação dos riscos por eles identificados como sendo os de maior probabilidade de manifestação no respetivo município de residência.

Palavras-chave: Perceção, riscos, educação, Área Metropolitana do Porto (AMP), Portugal.

\begin{abstract}
This study sets out to assess the perception of $9^{\text {th }}$ grade students about some fundamental concepts related to the science of risk and to ascertain their ability to identify and classify the natural and mixed risks most likely to occur, both at a national level and in Porto Metropolitan Area (PMA). A survey was carried out by means of a questionnaire and the results show that the students rated the risk of the occurrence of forest fire highest, not only nationally but also in all municipalities of the PMA, with a classification of fire occurrence ranging between high (nationally) and moderate (PMA municipalities). Some differences in the ranking defined for the different typologies of risk for the municipalities are noted. The study also suggests that most of the students have difficulty in identifying the origin, consequences and mitigation measures of the risks they identified as being the most likely to be found in the respective municipality of residence.
\end{abstract}

Keywords: Perception, risks, education, Porto Metropolitan Area (PMA), Portugal.

\footnotetext{
* O texto deste artigo corresponde a uma comunicação apresentada no IV Congresso Internacional de Riscos, tendo sido submetido em 24-10-2017, sujeito a revisão por pares a 26-10-2017 e aceite para publicação em 05-12-2017. Este artigo é parte integrante da Revista Territorium, $n .^{\circ} 25$ (II), 2018, ${ }^{\circ}$ RIscos, ISSN: 0872-8941.
} 


\section{Introdução}

De um modo mais ou menos generalizado, assiste-se ao crescente interesse pela temática dos riscos, devido ao aumento das suas manifestações, sobretudo a partir de meados da década de 50 do século passado. Os custos económicos e sociais delas decorrentes, não só relacionados com perdas humanas e danos económicos, mas também com os processos de recuperação/ reconstrução das áreas afetadas, são substanciais. Do mesmo modo, é reconhecido que a adopção de comportamentos adequados por parte da população, quer ao nível da prevenção, quer de gestão dos riscos naturais, poderá traduzir-se numa redução das consequências da manifestação dos riscos (Ardaya et al., 2017; Becker, et al., 2014).

Não negligenciando os factores associados à susceptibilidade, também a maior ou menor exposição das populações influencia significativamente a vulnerabilidade e, por conseguinte, as consequências da manifestação dos riscos (Nir, 1983; Panizza, 1990; Smith, 1996; Cunha e Cravidão, 2001; Cunha e Dimuccio, 2001, F. Rebelo, 2008; Nunes et al., 2015; Oliveira et al., 2017), razão pela qual o envolvimento da população é crucial para uma gestão mais eficaz dos riscos (Dixit, 2003).

A este respeito, vários autores sublinham que perspectivar as situações de crise somente como um problema técnico e ignorar a importância da participação das comunidades e, em especial, a forma como elas as percecionam é um erro em termos do processo de gestão das crises (Lebel, 2006, Birkholz et al., 2014; Bodoque et al., 2016). Com efeito, a perceção do público sobre os riscos é importante e, se ela for ignorada, pode resultar em designs de gestão tecnicamente fiáveis, mas inadequados a uma gestão eficaz (Granger-Morgan, 1997). Em termos práticos, a forma como as populações percepcionam os sinais de perigo é considerada um importante factor na determinação das suas respostas às medidas que são colocadas em ação no sentido de as proteger. Portanto, a compreensão do modo como os indivíduos percecionam o perigo que irão enfrentar irá influenciar cabalmente o grau de eficácia do design de gestão dos riscos naturais.

Uma perceção mais realista e acurada, por parte de uma população, acerca dos efeitos adversos dos riscos a que está sujeita, torna-a mais propensa e apta para adoptar comportamentos e estratégias pessoais que a protege dos danos, aceitando e, simultaneamente, apoiando iniciativas governamentais que sejam no sentido de prevenir e de remediar as consequências da manifestação de riscos (Slovic, 2000; Mcgee e Russell, 2003; Plapp e Werner, 2006). Simultaneamente, indivíduos classificados como mais conhecedores de um determinado risco são aqueles que têm mais consciência dos mesmos e evidenciam possuir estratégias mais eficazes de lidar com os riscos.

Por outro lado, alguns estudos revelam ainda que os indivíduos e as comunidades que experimentaram situações de crise estão mais propensas e motivadas para procurar mais informação sobre os riscos que as afetaram e a envolverem-se na resolução de problemas (Plapp e Werner, 2006, Miceli et al., 2008; Terpstra, 2009). Assim, a construção social do risco é apresentada, frequentemente, como uma consequência não só das formas de comunicação, nomeadamente condicionando os riscos que percepcionamos e a importância que thes atribuímos, mas também dependente das classes e das redes sociais em que os indivíduos se movem.

A sociedade atual, caraterizada como uma sociedade de conhecimento, ancorada em plataformas de inovação científica e tecnológica, requer dos cidadãos múltiplas formas de intervenção. Contudo, elas não parecem suficientes para conhecer e agir no paradigma da "sociedade de risco", uma vez que esta exige novas competências pessoais, fundadoras de uma cidadania mais ativa, participativa e informada, que devem ser adquiridas desde o início do percurso escolar.

A educação constitui, assim, uma das mais importantes e poderosas ferramentas na construção de novos conceitos, na mudança de hábitos e no diálogo intergeracional, desempenhando, por isso, um papel basilar e estruturante na implementação de mecanismos que conduzem a cidadãos melhor preparados e a sociedades mais resilientes, o que contribuirá para uma crescente consciencialização do risco e uma melhor perceção do perigo.

Assim, a campanha mundial sobre "A redução de catástrofes começa na escola”, desenvolvida em 2006 e 2007 pela Estratégia Internacional para Redução de Catástrofes EIRD/ONU, em resultado da Conferência Mundial sobre Redução de Catástrofes, realizada no Japão, em 2005, teve como objetivo informar e mobilizar os governos para que a redução de risco de catástrofes se integrasse nos currículos escolares, das escolas básicas e secundárias, de modo a aumentar a resiliência dos países face às catástrofes.

Em Portugal, só em 2011, o Conselho Nacional de Educação, através da Recomendação n. ${ }^{\circ}$ 5/2011, considerou que a escola deve promover a educação para o risco, inserindo nos seus curricula matérias relevantes sobre os diferentes conceitos de risco e tratando estas matérias não só pela transmissão de informação e conhecimentos, mas também promovendo a ação que, na prática, lida com casos específicos de risco. Deste modo, pretende "transformar a escola num agente de intervenção e num motor de mobilização da sociedade, em matéria de Educação para o Risco, através dos alunos, das suas famílias e da restante comunidade educativa" (Nunes et al., 2013). 
A Geografia, não só como disciplina integradora, mas também porque procura responder às questões que o Homem coloca sobre o meio físico e humano, os quais interagem entre si e se alteram constantemente, pode desempenhar um papel fulcral na "educação para o risco". Com efeito, o conhecimento e a compreensão da sociedade e do planeta em que vivemos podem constituir instrumentos essenciais na formação e na informação dos futuros cidadãos, ou seja, na promoção de uma cidadania interventiva (Silva e Ferreira, 2000).

Nessa perspectiva, as orientações curriculares propostas pela reforma de 2001 introduziram, no 7. ${ }^{\circ}$ ano de escolaridade, o subtema 'Riscos e Catástrofes Naturais' o qual se integra no tema 'Meio Natural' e onde são desenvolvidos conteúdos relacionados com as "Causas das catástrofes naturais" e os "Efeitos sobre o homem e sobre o ambiente". Todavia, as indicações emanadas pelas orientações curriculares pareciam manifestamente insuficientes, pois apenas eram no sentido de abordar as causas e os efeitos das catástrofes, sem qualquer referência à explicitação dos conceitos básicos, como os de risco e catástrofe, ou a definição dos diferentes tipos de risco. Por outro lado, o processo de ensino/aprendizagem deve centrar-se na prevenção e mitigação das catástrofes, aspetos importantes para a criação de uma cultura de prevenção, que pode levar à redução dos efeitos negativos das suas consequências (Tedim et al.,2010).

No intuito de colmatar estas deficiências e através das metas curriculares, numa iniciativa do Ministério da Educação e Ciência, implementou-se no 9. ${ }^{\circ}$ ano de escolaridade o domínio "Riscos, Ambiente e Sociedade", tendo como objetivo inicial a compreensão/explicitação de um conjunto de conceitos relacionados com a teoria do risco, tais como: suscetibilidade, vulnerabilidade, risco e catástrofe. A identificação de diferentes riscos quanto às suas causas (naturais, antrópicos e mistos) constituiu outro dos descritores enunciados.

Assim, os riscos naturais enunciados são, essencialmente, de cariz climático/meteorológico (furacões, tornados e tempestades de vento, secas, ondas de calor e vagas de frio), hidrológico (cheias e inundações) e geomorfológico (movimentos de vertente). Já no que se refere aos riscos mistos, privilegiaram-se os que, devido à ação antrópica, se desenvolvem no seio da atmosfera (formação do smog, chuvas ácidas, aumento dos gases com efeito de estufa e destruição da camada do ozono) e da hidrosfera (degradação das águas continentais e marinhas), assim como os que afetam a litosfera e a biosfera (erosão e degradação do solo, desertificação e incêndios florestais). A implementação curricular destas metas viria a ocorrer apenas no ano letivo de 2015/16.

Por sua vez, em 2015 foi apresentado o "Referencial de Educação para o Risco (RERisco)", um trabalho conjunto da Direção-Geral da Educação (DGE), Direção-Geral dos Estabelecimentos Escolares (DGEstE) e Autoridade Nacional de Proteção Civil (ANPC), destinado à Educação Pré-Escolar, ao Ensino Básico e ao Ensino Secundário, o qual se propõe contribuir para a concretização da educação para o risco, no quadro da Educação para a Cidadania, tanto na sua dimensão transversal, como no desenvolvimento de projetos e iniciativas que contribuam para a formação pessoal e social dos alunos e, ainda, na oferta de componentes curriculares complementares, nos $1 .^{\circ}, 2 .^{\circ}$ e $3 .^{\circ}$ ciclos do ensino básico.

Embora, no nosso país, a preocupação com a "Educação para os riscos", em ambiente escolar, se encontre numa fase "embrionária”, com o presente estudo procurámos avaliar:

(i) a perceção que os alunos do $9^{\circ}$ ano letivo têm sobre conceitos fundamentais associados à ciência do risco (tais como: risco, catástrofe, susceptibilidade e vulnerabilidade);

(ii) a dimensão espacial que os alunos têm dos riscos naturais e mistos que afetam tanto o território continental, como a respectiva área de residência, neste caso, a Área Metropolitana do Porto (AMP);

(iii) se os discentes são capazes de identificar as causas, consequências e medidas de mitigação para o risco que consideram ter maior probabilidade de ser manifestar na respetiva área de residência.

Além disso, de acordo com o National Research Council (NRC), com este exercício pretendeu-se analisar também o pensamento espacial destes discentes, o qual tem o objectivo educacional de não só promover o hábito de pensar espacialmente, mas também fomentar a prática do pensamento espacial de uma forma informada e, ainda, a adopção de uma postura crítica relativamente ao pensamento espacial (NRC, 2006).

\section{Metodologia}

Com o intuito de aferir as percepções que os alunos do $9^{\circ}$ ano de escolaridade têm dos conceitos fundamentais associados à "Ciência do Risco" (tais como: risco, catástrofe, suscetibilidade e vulnerabilidade), bem como de avaliar a respetiva capacidade em identificar e classificar os riscos, naturais e mistos, com maior probabilidade de se manifestarem, tanto a nível nacional como à escala da AMP, procedeu-se à aplicação de um inquérito por questionário antes do tema ser lecionado no âmbito da disciplina de Geografia, cujos conteúdos se integram no Domínio "Riscos, Ambiente e Sociedade”.

0 questionário foi aplicado a um total de 308 alunos do $9^{\circ}$ ano de escolaridade $\left(3^{\circ}\right.$ ciclo), com idades compreendidas entre 14 e 16 anos que integram escolas da AMP, mais especificamente os concelhos de Matosinhos, Porto, Vila Nova de Gaia e Gondomar. A sua implementação ocorreu durante o mês de abril de 2017, antes do referido domínio ter sido lecionado. 
Este questionário divide-se em 3 partes. A primeira delas procura avaliar os conhecimentos que os alunos do $9^{\circ}$ ano de escolaridade têm sobre conceitos fundamentais associados à "ciência do risco", designadamente os de risco, susceptibilidade, vulnerabilidade e catástrofe. Para cada um destes conceitos foram colocadas 4 opções de resposta, tendo-se pedido para identificar a que consideravam correta. A escolha destes conceitos deve-se à sua importância no modelo conceptual-operacional risco-perigo-crise. 0 risco corresponde a um "sistema complexo de processos cuja modificação de funcionamento é susceptível de acarretar prejuízos diretos ou indiretos (perda de recursos) a uma dada população" (Faugères, 1990), o que comporta os processos inerentes à manifestação de um determinado risco (Rebelo, 1995), bem como a incerteza que decorre do que é suscetível. Comporta, ainda, as consequências, plasmadas nos prejuízos que o risco comporta para uma dada população e que, naturalmente, serão maiores ou menores em função da vulnerabilidade desta (Almeida, 2011). A catástrofe corresponde a acontecimentos súbitos ou séries de acontecimentos graves, quase sempre imprevisíveis, de origem natural ou antrópica, com efeitos prolongados no tempo e no espaço, suscetíveis de provocarem elevados prejuízos materiais e, eventualmente, vitimas, afetando gravemente a segurança das pessoas, as condições de vida das populações e o tecido socioeconómico dum país, em áreas extensas do seu território, fechando deste modo um modelo conceptual-operacional risco-perigo-crise

$\mathrm{Na}$ segunda parte do questionário, pretende-se avaliar a capacidade dos discentes identificarem e classificarem a probabilidade de ocorrência de riscos naturais e mistos à escala nacional e no concelho de residência. Para esse feito, foram elencados 17 riscos naturais e mistos e, para os escalonar, introduziu-se uma escala qualitativa, variável entre um valor nulo/mínimo e um valor máximo, a que se fez corresponder uma escala quantitativa, respetivamente ordenada de 1 a 5, como se indica: 1 - nulo/mínimo; 2 - reduzido; 3 - moderado; 4 - elevado; 5 - máximo. Neste sentido, o valor mais baixo (nulo ou mínimo) está associado a uma perceção do risco muito baixa em função da probabilidade de manifestação do risco, em oposição ao valor mais elevado (máximo), associada a uma probabilidade de manifestação muito alta.

Na sequência, procedeu-se à normalização dos valores obtidos, quer a nível nacional quer a nível municipal, tendo sido atribuído 100 ao valor total. Os restantes valores foram normalizados em função desse valor total $(=100)$, o que permite hierarquizar os riscos em função da respetiva probabilidade de manifestação.

Depois, para detetar grupos homogéneos nos dados recolhidos, aplicou-se uma análise de Clusters, ou seja, um procedimento multivariado (Pestana e Gageiros, 1998). De facto, a Classificação Hierárquica de Clusters é um dos métodos utilizados e serve, essencialmente, para medir a hierarquia da "proximidade" entre objetos que, neste caso, são os riscos.

Para o efeito, utilizou-se método average linkage ou ligação por média. Algumas características desse método são: menor sensibilidade a ruídos, face aos métodos de ligação por vizinho mais próximo e por vizinho mais distante; apresentação de bons resultados, tanto para distâncias Euclidianas como para outras distâncias; tendência a formar grupos com número de elementos similares (Kaufmann, 1990). Deste modo, o dendrograma é a expressa gráfica da matriz de proximidade entre as amostras, uma vez que hierarquiza a similaridade ou dissimilaridade dos riscos percepcionados pelos alunos, quer a nível nacional, quer no respetivo concelho de residência.

Posteriormente, com o intuito de aferir o grau da correlação linear entre os resultados obtidos tanto a nível nacional como entre os vários concelhos, aplicouse o coeficiente de correlação de Pearson ( $r$ ), um índice adimensional com valores situados ente $-1,0$ e 1,0 inclusive, que reflete a intensidade da relação linear entre dois conjuntos de dados.

Por último, apresentam-se os resultados da terceira parte do questionário, onde foram colocadas 3 questões com o intuito de aferir o conhecimento dos alunos no que respeita às causas, consequências e medidas de mitigação do risco que consideraram mais elevado em função da sua manifestação no concelho de residência.

\section{Resultados}

\section{Conceitos fundamentais associados à ciência do risco}

Um pouco mais de metade dos alunos inquiridos (51,3\%) respondeu corretamente quando thes foi solicitado para identificarem o conceito de risco, que entendem como sendo a "combinação da probabilidade de ocorrência de um evento potencialmente perigoso com as suas consequências negativas". As restantes opções, "acontecimento com efeitos relativamente limitados no tempo e no espaço, susceptíveis de causar danos em pessoas e bens"; "manifestação de fenómenos físicos, tecnológicos e antrópicos e respectivas consequências sobre pessoas $e$ bens" $e$ "consequências físicas e humanas resultantes da ocorrência de fenómenos perigosos" colheram uma percentagem de respostas muito semelhante, próxima de 15\% (fig. 1a).

No que concerne ao conceito de "Catástrofe", a opção correta "plena manifestação do risco e suas consequências" obteve apenas $17,2 \%$ das respostas, valor significativamente inferior ao de $46,9 \%$, obtido quando se considera a catástrofe como "grande calamidade, devida às elevadas perdas humanas e materiais", o que certamente decorre do facto dos alunos considerarem apenas a parte mais visível das catástrofes e que, naturalmente, são as suas graves consequências. 


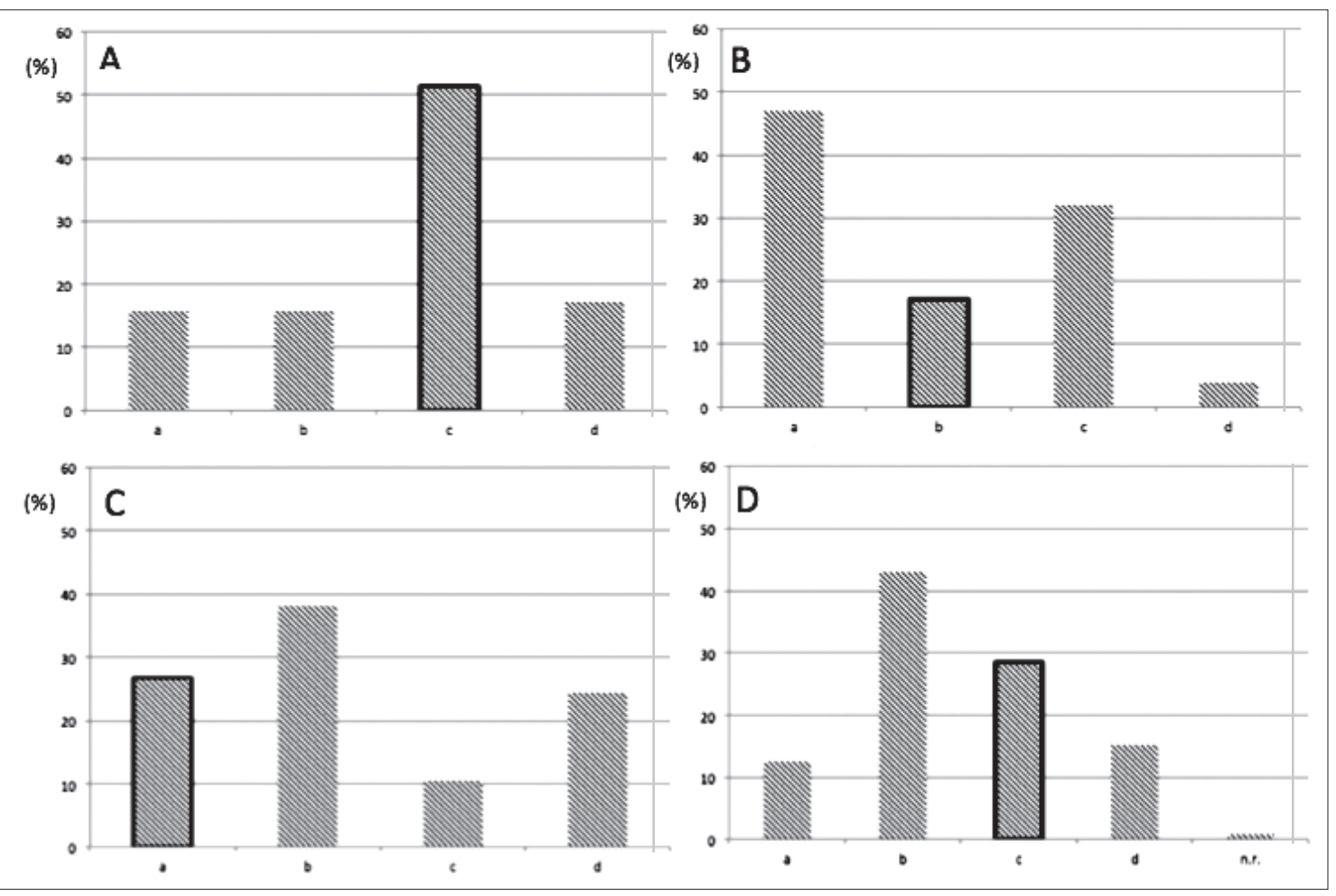

Fig. 1 - Distribuição (em \%) das respostas aos conceitos de Risco (a), Catástrofe (b), Susceptibilidade (c) e Vulnerabilidade (d).

Fig. 1 - Distribution (in \%) of the responses to the concepts of risk (a), Disaster (b), Susceptibility (c) and Vulnerability (d).

Dentro desta mesma linha de pensamento, cerca de $30 \%$ compreende que uma catástrofe corresponde aos "prejuízos que um fenómeno natural acarreta para uma dada população". Os restantes 3,9\% entendem-na como sendo um "acontecimento que resulta do não controlo de produtos químicos perigosos" (fig.1b).

Relativamente ao conceito de "susceptibilidade", ele foi preferencialmente entendido, por cerca de $38 \%$ dos alunos, como a "propensão de uma área ser afetada por uma catástrofe natural”. A resposta correta, "condições que um território apresenta para a ocorrência de um fenómeno destrutivo", obteve um valor ligeiramente inferior, $27 \%$ das respostas, provavelmente devido ao facto das duas respostas apresentarem alguma semelhança, situação que merece ser corrigida em próximas passagens de inquéritos. A opção "elementos naturais e humanos expostos a um determinado risco" apresentou um valor muito próximo do obtido na resposta anterior, ou seja, $24,4 \%$. Os restantes $10,0 \%$ entende a susceptibilidade como correspondendo a uma “área geográfica de risco elevado" (fig. 1c).

Por sua vez, o conceito de "vulnerabilidade" foi entendido, por $43 \%$ dos discentes, como sendo a "área geográfica mais exposta a um risco natural ou antrópico", valor significativamente superior à opção correta, "caraterísticas e circunstâncias de uma comunidade que a tornam suscetível aos efeitos nocivos do processo", que obteve um total de respostas ligeiramente inferior a $30 \%$. As opções "capacidade de resposta da população afectada por fenómeno perigoso" e "medidas de proteção experimentadas antes da ocorrência de um fenómeno natural, tecnológico ou antrópico" colheram, respetivamente, $15,2 \%$ e $12,5 \%$ das respostas (fig. $1 \mathrm{~d}$ ).

\section{Perceção a nível nacional e municipal (AMP)}

A sistematização da distribuição qualitativa e quantitativa da probabilidade de manifestação dos diferentes riscos percecionada pelos alunos do $9^{\circ}$ ano de escolaridade, respetivamente, no território nacional e na AMP (fig. 2 - A e B; QuADRos I e II) permitiu retirar várias conclusões, de que se salientam as seguintes:

De um modo geral, a perceção que os alunos têm da dimensão espacial dos riscos naturais e mistos, em função da sua probabilidade de ocorrência, oscilou entre reduzida e moderada, para cerca de $60 \%$ dos inquiridos, quando tratada à escala nacional, e variou entre mínima e reduzida, para mais de $65 \%$ dos respondentes, quando se considerou apenas a AMP.

Com uma probabilidade de ocorrência mínima a reduzida, no espaço nacional, emergiram os riscos geofísicos, que incluem o risco vulcânico e de tsunami, e alguns dos meteorológicos, nomeadamente a ocorrência de furacões e tornados. A perceção que os alunos tinham 
relativamente ao risco de desertificação foi, igualmente, de probabilidade de manifestação mínima/nula a reduzida (fig. 2A).

Depois, com uma classificação preponderante nas classes de manifestação reduzida-moderada surgem o risco sísmico, as secas, as ondas de frio, a degradação dos solos e os movimentos de vertente. Nas categorias subsequentes, de moderado a elevado destacam-se, por congregarem entre 60 a 70\% das respostas, as ondas de calor, as cheias e as inundações, a poluição da água e do ar e a erosão costeira. Os incêndios florestais são os que assinalam maiores frequências relativas, num total de $75 \%$, nas classes de maior probabilidade de manifestação, ou seja, nas classes de elevada a máxima manifestação (fig. 2A).
No que se refere à AMP, as classes que registaram maiores frequências relativas acumuladas foram, em termos médios, a mínima e a reduzida. No caso dos riscos geofísicos, os valores oscilaram entre 88 e $96 \%$. Com valores praticamente idênticos afiguraramse também os furacões e os tornados. Por sua vez, a ocorrência de secas, os movimentos de vertente e os processos de desertificação foram percecionados pela maioria dos discentes como sendo de risco nulo/mínimo a reduzido. Por fim, a manifestação dos restantes riscos naturais e mistos identificados emergiram com uma maior probabilidade de manifestação, embora a maior percentagem de frequências se integre nas classes de reduzido a moderado. Apenas se destacam os incêndios florestais, em que $23 \%$ dos alunos classificam como elevada a probabilidade de ocorrerem na AMP (fig. 2B).
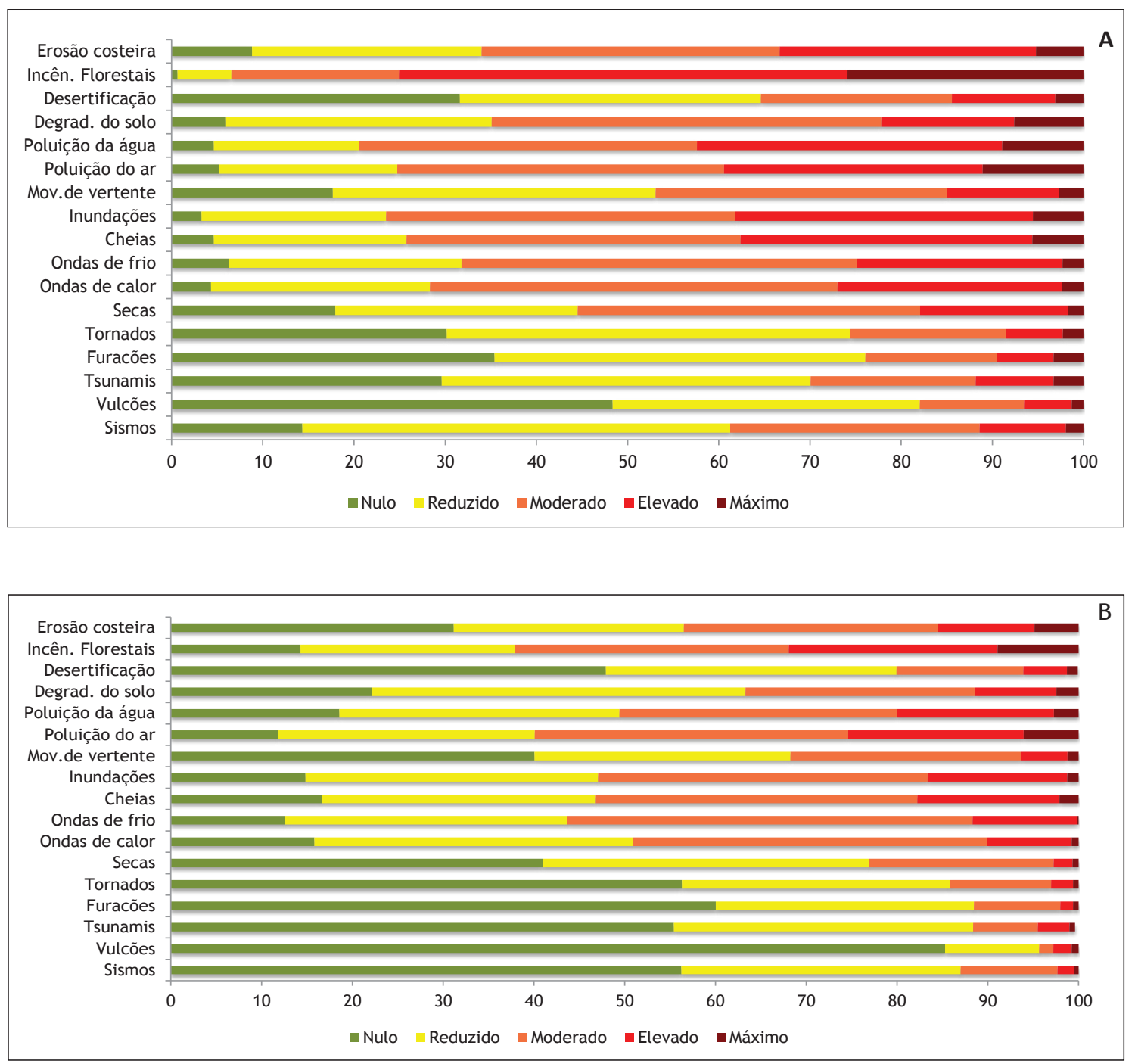

Fig. 2 - Classificação dos riscos em função da sua probabilidade de ocorrência no território nacional (A) e na AMP (B), de acordo com a perceção dos alunos de $9 .^{\circ}$ ano de escolaridade.

Fig. 2 - Classification of risks relative to probability of occurrence in the national territory (A) and in the PMA (B), according to the $9^{\text {th }}$ grade students' perception. 
QUADRo I - Distribuição das frequências relativas (\%) por classes de manifestação dos diferentes riscos, a nível nacional e na AMP (a sombreado as classes com maior frequência relativa).

$T_{A B L E}$ I - Distribution of relative frequency (\%) by the manifestation ranking of different risks at national level and in the PMA (highest relative frequency shaded light gray).

\begin{tabular}{|l|c|c|c|c|c|c|c|c|c|c|}
\cline { 2 - 11 } \multicolumn{1}{|c|}{} & \multicolumn{9}{|c|}{ NACIONAL } & \multicolumn{3}{c|}{ AMP } \\
\hline & Nulo & Reduzido & Moderado & Elevado & Máximo & Nulo & Reduzido & Moderado & Elevado & Máximo \\
\hline Sismos & 14,3 & 46,9 & 27,4 & 9,4 & 2,0 & 56,2 & 30,8 & 10,7 & 1,8 & 0,5 \\
\hline Vulcões & 48,4 & 33,7 & 11,4 & 5,2 & 1,3 & 85,3 & 10,4 & 1,6 & 2,0 & 0,7 \\
\hline Tsunamis & 29,6 & 40,5 & 18,1 & 8,6 & 3,3 & 55,4 & 33,0 & 7,1 & 3,5 & 0,6 \\
\hline Furacões & 35,4 & 40,7 & 14,4 & 6,2 & 3,3 & 60,0 & 28,4 & 9,5 & 1,4 & 0,6 \\
\hline Tornados & 30,2 & 44,3 & 17,0 & 6,2 & 2,3 & 56,3 & 29,5 & 11,2 & 2,4 & 0,6 \\
\hline Secas & 17,9 & 26,6 & 37,5 & 16,3 & 1,7 & 41,0 & 36,0 & 20,3 & 2,1 & 0,7 \\
\hline Ondas de calor & 4,3 & 24,0 & 44,7 & 24,7 & 2,3 & 15,8 & 35,1 & 39,0 & 9,3 & 0,7 \\
\hline Ondas de frio & 6,3 & 25,5 & 43,4 & 22,5 & 2,3 & 12,6 & 31,1 & 44,7 & 11,5 & 0,2 \\
\hline Cheias & 4,6 & 21,1 & 36,6 & 32,0 & 5,6 & 16,6 & 30,2 & 35,4 & 15,6 & 2,1 \\
\hline Inundações & 3,3 & 20,3 & 38,2 & 32,7 & 5,6 & 14,8 & 32,3 & 36,3 & 15,4 & 1,3 \\
\hline Mov. de vertente & 17,7 & 35,4 & 32,0 & 12,2 & 2,7 & 40,1 & 28,2 & 25,4 & 5,1 & 1,2 \\
\hline Poluição do ar & 5,2 & 19,5 & 35,8 & 28,3 & 11,1 & 11,8 & 28,3 & 34,5 & 19,3 & 6,1 \\
\hline Poluição da água & 4,6 & 15,9 & 37,1 & 33,4 & 8,9 & 18,6 & 30,9 & 30,6 & 17,3 & 2,7 \\
\hline Degrad. do solo & 6,0 & 29,1 & 42,7 & 14,6 & 7,6 & 22,1 & 41,2 & 25,3 & 9,0 & 2,4 \\
\hline Desertificação & 31,6 & 33,0 & 21,0 & 11,3 & 3,1 & 47,9 & 32,0 & 14,0 & 4,8 & 1,2 \\
\hline Incên. florestais & 0,7 & 5,9 & 18,4 & 49,2 & 25,9 & 14,3 & 23,6 & 30,2 & 23,0 & 8,9 \\
\hline Erosão costeira & 8,8 & 25,2 & 32,7 & 28,1 & 5,2 & 31,2 & 25,3 & 28,0 & 10,6 & 4,9 \\
\hline Média & 15,8 & 28,7 & 29,9 & 20,1 & 5,5 & 35,3 & 29,8 & 23,7 & 9,1 & 2,1 \\
\hline
\end{tabular}

\section{“Ranking” espacial dos riscos}

A quantificação da perceção que os alunos têm da espacialização dos diferentes tipos de riscos permitiu hierarquizar e identificar de forma mais específica a avaliação dos riscos em função da sua manifestação, quer à escala nacional quer no respetivo município de residência.

A nível nacional destaca-se o risco de incêndios florestais. Com efeito, no panorama nacional, os incêndios florestais assumem uma manifestação recorrente, sobretudo no Norte e Centro de Portugal, amplamente divulgados pelos meios de comunicação, tornando-os, naturalmente, num risco com elevada probabilidade de ocorrência (fig. 3).

Todavia, a nível municipal, só surgem em primeiro lugar nos concelhos de Gondomar e Vila Nova de Gaia. Em Matosinhos, assumem a segundo posição, sendo que os alunos identificam a poluição do ar como o risco com maior probabilidade de manifestação. Por sua vez, no município do Porto, as cheias e as inundações são os riscos que causam maior preocupação aos discentes, colocando-os, assim, no topo em termos de manifestação, o que não surpreende, já que sendo um município quase exclusivamente urbano, praticamente não regista incêndios florestais.

Por outro lado, a nível nacional, destacam-se também os riscos de poluição da água e do ar, a par das inundações/ cheias, por terem sido percecionados como sendo os de risco mais elevado, em função da sua manifestação. Mas, em contrapartida, nos munícipios de Gondomar e Vila Nova de Gaia são os riscos de ondas de calor e de frio, aqueles que se posicionam nas posições cimeiras, enquanto que nos municipio do Porto e de Matosinhos se destaca o risco a erosão costeira.

Por último, o risco de vulcanismo foi percecionado, quer a nível nacional quer à escala municipal, como um risco reduzido, ocupando a última posição do "ranking”, a que se seguem, de um modo geral, os sismos, furacões, tornados e tsunamis, embora com diferentes posições (fig. 3).

Similaridade e correlações entre as escalas nacional e municipal

A aplicação da análise hierárquica de Clusters à perceção que alunos têm da espacialização dos diferentes tipos de riscos mostra que a nível nacional eles individualizaram a ocorrência de incêndios florestais, face à probabilidade de manifestação dos restantes riscos. Com certeza que a intensa manifestação deste risco, amplamente divulgada pelos meios de comunicação, explicará esta perceção (fig. 4).

Num segundo grupo, individualizam-se aqueles riscos que foram percecionados como tendo uma manifestação reduzida e que correspondem aos riscos de: furacões, tornados, tsunamis, desertificação e vulcanismo. 

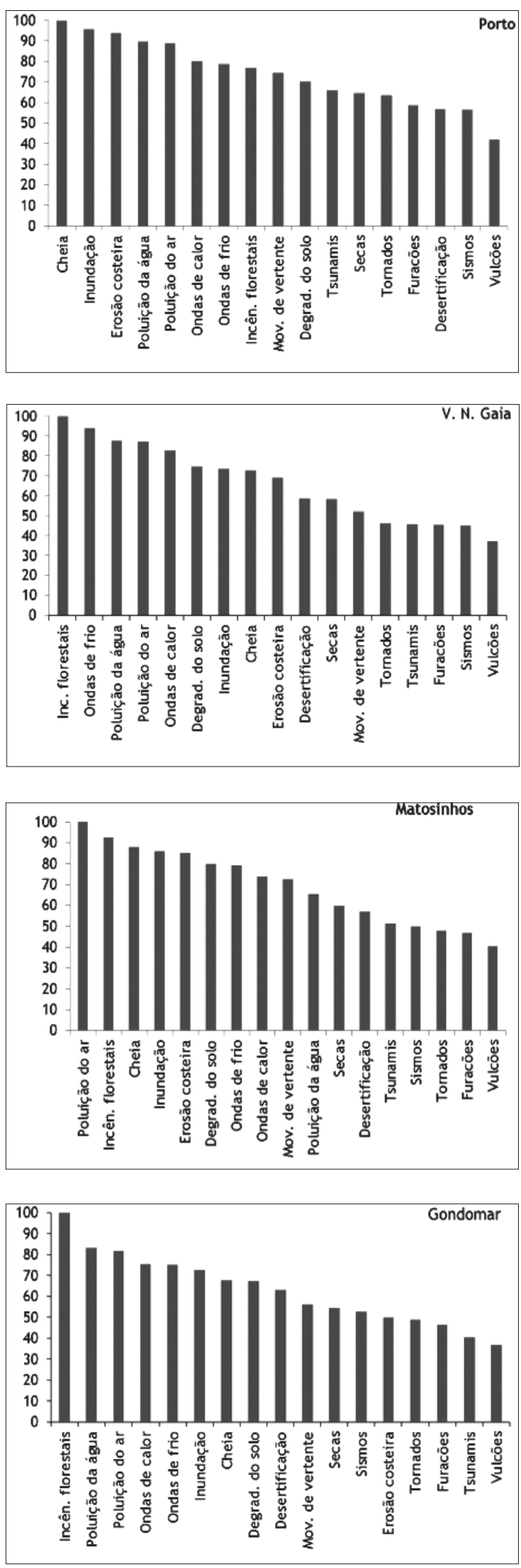

Fig. 3 - Ranking dos riscos analisados, para a escala municipal, de acordo com a perceção dos alunos.

Fig. 3 - Ranking of the risks analyzed, for the municipal level, according to the students' perception.
Num terceiro Cluster integram-se os restantes riscos, divididos em 2 subgrupos: um deles é constituído por cheias, inundações, poluição da água, poluição do ar e erosão costeira, a par das ondas de frio, de calor e degradação dos solos; o outro é formado por secas, movimentos de vertente e sismos.

A nível municipal, os resultados obtidos mostram ligeiras similaridades e discrepâncias, entre si e em relação à realidade nacional, pelo que merecem ser destacadas (fig. 5). Assim, em nenhum destes municípios o risco de incêndios florestais se individualiza enquanto Cluster, ao invés da perceção à escala nacional. Com efeito, foi incluído no cluster de outros riscos com maior potencial de ocorrência, por conseguinte, a par do risco de poluição do ar e da água.

Deste modo, os alunos dos municípios do Porto e de Vila Nova de Gaia individualizaram os vulcões como sendo um risco com nula probabilidade de ocorrência, incluindo os restantes em 2 subgrupos principais. Embora a sequência não seja a mesma, a similitude na agregação dos riscos é bastante idêntica, apenas diferindo a erosão costeira, que é percecionada como mais relevantes no município do Porto face ao de Vila Nova de Gaia, ao contrário do que se passa com os movimentos de vertente que foram percecionados como mais importantes no concelho de Vila Nova de Gaia quando comparado com o do Porto (fig. $5 \mathrm{a}$, b).

A aplicação da análise hierárquica de Clusters aos municípios de Matosinhos e Gondomar faz emergir dois conjuntos principais de riscos, os quais agregam os riscos que se relacionam mais fortemente entre si. Assim, os resultados apurados para Matosinhos e Gondomar apenas diferem na classificação da poluição da água e dos movimentos de massa, integrados no grupo de riscos com menor probabilidade de ocorrerem, respetivamente, em Matosinhos e Gondomar (fig. 5 c, d).

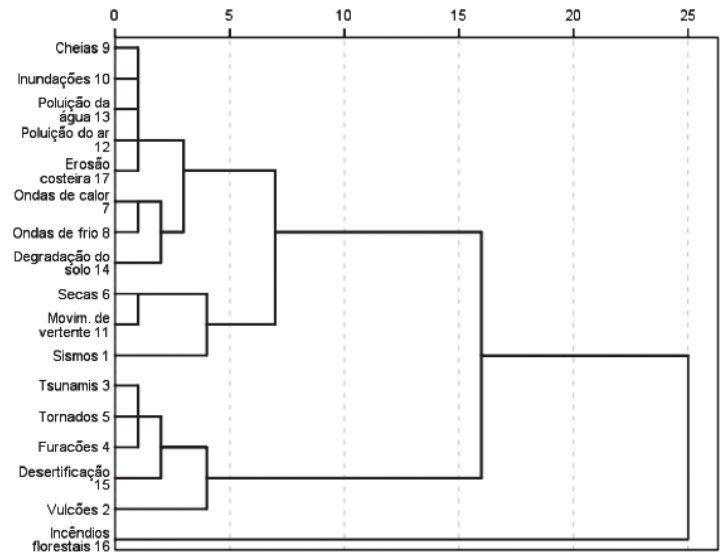

Fig. 4 - Análise hierárquica de Clusters relativa à manifestação de diferentes riscos, a nível nacional.

Fig. 4 - Hierarchical analysis of clusters regarding the manifestation of different risks, at national level. 

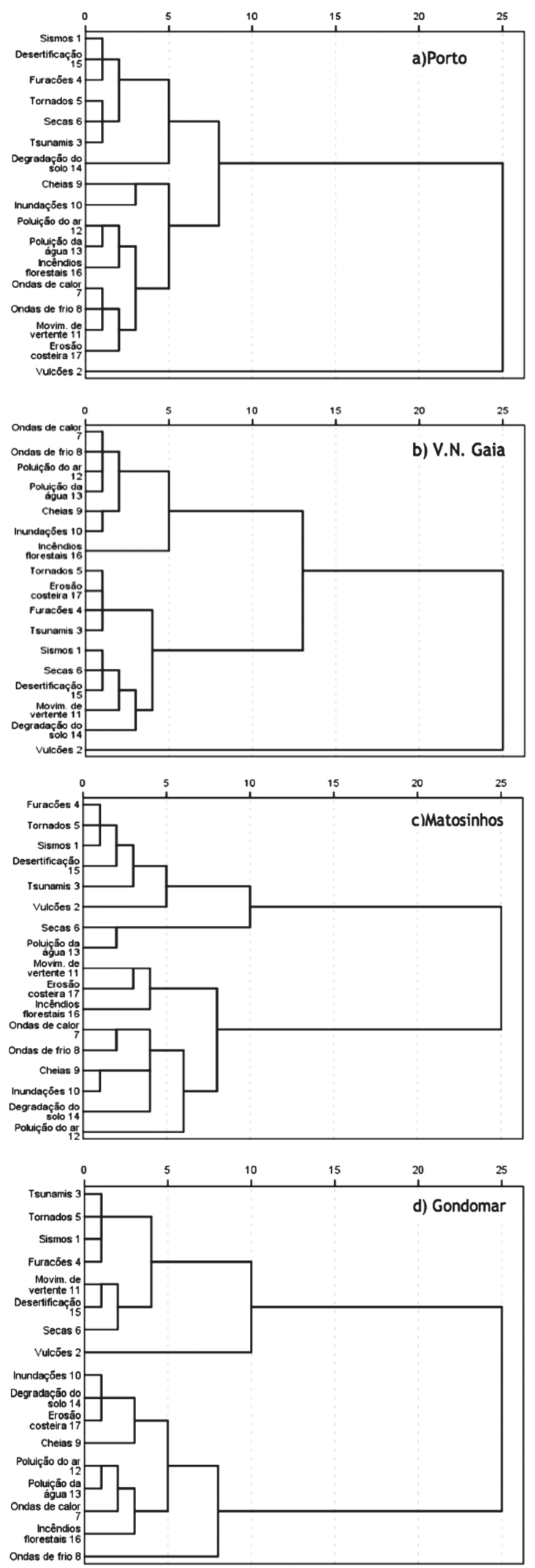

Fig. 5 - Análise hierárquica de Clusters referentes à manifestação de diferentes riscos, a nível municipal.

Fig. 5 - Hierarchical analysis of clusters related to the manifestation of different risks, at municipal level.
A sistematização das correlações obtidas através da aplicação do coeficiente de Pearson, cujo intuito é aferir o grau de associação que os alunos têm relativamente à probabilidade de manifestação do conjunto de riscos analisados, à escala nacional e no município de residência, apresentou como resultado mais significativo e que denota uma perceção muito idêntica entre a probabilidade de manifestação das várias tipologias de riscos abordados, a correlação existente entre os resultados a nível nacional e o município de Vila Nova de Gaia (r: 0,910). Em contrapartida, o menor coeficiente $(r: 0,517)$ é o que resulta da correlação entre os resultados obtidos a nível nacional e no município do Porto.

Por sua vez, ao considerar a classificação da probabilidade de manifestação dos riscos naturais e mistos entre os municípios estudados, observa-se que nos concelhos de Vila Nova de Gaia e Gondomar ela é praticamente idêntica, uma vez que o respectivo coeficiente de correlação é de 0,929 (QUADRo III).

Quadro II - Correlações de Pearson entre a classificação dos riscos a nível nacional e entre os municípios em estudo.

TABLE II - Pearson's correlations between the risk ranking at national level and between the municipalities studied.

\begin{tabular}{|l|c|c|c|c|c|}
\cline { 2 - 6 } \multicolumn{1}{c|}{} & Nacional & Porto & V. N. Gaia & Matosinhos & Gondomar \\
\hline Nacional & 1 & & & & \\
\hline Porto & 0,517 & 1 & & & \\
\hline V. N. Gaia & 0,910 & 0,694 & 1 & & \\
\hline Matosinhos & 0,881 & 0,829 & 0,824 & 1 & \\
\hline Gondomar & 0,894 & 0,580 & 0,929 & 0,746 & 1 \\
\hline
\end{tabular}

Causas, consequências e medidas de mitigação: o que sabem os alunos?

A análise dos resultados obtidos na terceira parte do questionário, a qual se refere ao conhecimento que os alunos têm face às causas, consequências e medidas de mitigação do risco que consideram ser o mais elevado, face ao número de ocorrências com que se manifesta, sugerem que os alunos possuem muitas dificuldades no que respeita ao conhecimento dos fatores que estão na génese do risco, bem como, nas formas de os mitigar. Cerca de $70 \%$ dos alunos não respondeu corretamente às três questões colocadas. Destas, destacam-se as "causas" e as "consequências" como aquelas com maior número de respostas incorretas, ambas com valores muito próximo de $75 \%$. No que respeita às medidas de mitigação, cerca de $42 \%$ elencou medidas corretas consonante com o risco identificado (QuadRo III). Para o risco de incêndios, as causas atribuídas foram a "falta de limpeza das florestas" e o "incendiarismo". As consequências apontadas foram a "destruição florestal" 
e a "perda de habitações e terrenos agrícolas". No que respeita às medidas de mitigação, destacamse a "existência de guardas florestais", "menos lixo das florestas" e "limpeza das florestas", bem como, "punição severa para os incendiários". No que respeita ao segundo risco mais considerado, a poluição da água,

"não poluir" foi a medida mais considerada como forma de mitigação, cerca de 63\%, seguida de "legislar e punir quem polui" (14\%), "construção de ETAR" (11\%), "inspecionar os emissores poluentes" (7\%). Para o risco de poluição do ar foram varias as medidas de mitigação consideradas. "Utilizar menos transportes e andar mais a pé" foi a medida mais considerada, cerca de metade, seguido da "utilização de transportes públicos", "utilização de energias renováveis", "uso de carros elétricos", "reduzir as fontes de poluição", "reduzir o número de fábricas", "relocalizar as fábricas para longe das pessoas", "promover a reciclagem", "mais espaços verdes" e "diminuir os gases com efeito de estufa". No que respeita ao risco de cheias e inundações, a "construção de estruturas de proteção como paredões" colheu cerca de $30 \%$ das respostas, seguido de "não construir em locais de risco elevado", $23 \%$, "melhorar o sistema de saneamento", $8 \%$, "diminuir a poluição", "construção de barragens", "aumentar a permeabilização do solo", "reciclar” e "educar a população e instruir para situações de crise", estas últimas com valores inferiores a $5 \%$.

De modo geral, detetou-se também uma relação entre a dificuldade em responder às questões relacionadas com a génese, consequências e medidas de mitigação do risco natural e o conhecimento dos conceitos de risco, catástrofe, suscetibilidade e vulnerabilidade. Com efeito, considerando os cerca de $70 \%$ de questionários em que os alunos não responderam corretamente às questões, verifica-se que em apenas 39\% deles responderam corretamente ao conceito de risco. Essa discrepância é menos observável no que respeita aos restantes conceitos.

QUADRo III - Análise às respostas relativas ao conhecimento das causas, consequências e medidas de mitigação do risco considerado, em função da probabilidade de se manifestar como mais elevado, no concelho de residência.

TABLE III - Analysis of responses related to knowledge of the causes, consequences and mitigation measures of the risk considered, according to the highest probability of it being manifested in the municipality of residence.

\begin{tabular}{|c|c|c|c|c|c|}
\hline \multicolumn{2}{|c|}{$\begin{array}{c}\text { Identifica } \\
\text { causas }\end{array}$} & \multicolumn{2}{c|}{$\begin{array}{c}\text { Identifica } \\
\text { consequências }\end{array}$} & \multicolumn{2}{c|}{$\begin{array}{c}\text { Indica medidas } \\
\text { de mitigação }\end{array}$} \\
\hline Sim & Não & Sim & Não & Sim & Não \\
$(\%)$ & $(\%)$ & $(\%)$ & $(\%)$ & $(\%)$ & $(\%)$ \\
\hline 25 & 75 & 22 & 78 & 42 & 58 \\
\hline
\end{tabular}

\section{Considerações finais}

A perceção que os alunos têm dos riscos, da sua intensidade e da probabilidade da sua manifestação, é essencial na educação para o risco. Em termos conceptuais, assinalou-se alguma confusão em termos de terminologia, sobretudo quando foram avaliados conceitos, como os de susceptibilidade, vulnerabilidade e catástrofe.

Essa dificuldade foi ainda mais vincada nos alunos que, em elevado número, não conseguiram identificar as causas, as consequencias e as medidas de mitigação adequadas para o risco que, em função da probabilidade em se manifestar consideram ser o mais importante na concelho de residência.

Apesar de, modo geral, os alunos terem identificado os riscos que apresentam maior probabilidade de se manifestarem, quer a nível nacional, quer na respetiva área de residência, evidenciaram arduidade na identificação dos principais fatores que estão na génese dos riscos, bem como nas respectivas consequências e, ainda, nas medidas de mitigação. Salienta-se, a atribuição, a nível local, de uma probabilidade de manifestação mínima a reduzida à generalidade dos riscos, com exceção dos incêndios florestais, poluição da água e do ar, cheias e inundações. Por outro lado, a probabilidade de ocorrência que atribuíram à sua área de residência foi, num análise comparativa, sempre inferior à referida na escala nacional.

\section{Bibliografia}

Almeida, A. Betâmio de (2011). Risco e gestão do risco. Questões filosóficas subjacentes ao modelo técnico conceptual. Territorium 18, p. 23-31. Disponível em: http://www.uc.pt/fluc/nicif/riscos/Documentacao/ Territorium/T18_artg/Antonio_Betamio_de_Almeida.pdf

Ardaya, A., Evers, M., Ribbe, L. (2017). What influences disaster risk perception? Intervention measures, flood and landslide risk perception of the population living in flood risk areas in Rio de Janeiro state, Brazil, International Journal of Disaster Risk Reduction, 25, p.227-237.

Becker, G., J. Aerts, J., Huitema, D. (2014). Influence of flood risk perception and other factor on risk reducing behaviour: a survey of municipalities along the Rhine, J. Flood Risk Manag. 7 (1) p.16-30. DOI: http://dx.doi.org/10.1111/jfr3.12025

Birkholz, S., Muro, M., Jeffrey, P., Smith, H. (2014). Rethinking the relationship between flood risk perception and flood management, Science of The Total Environment, 478, p. 12-20.

DOI:http://dx.doi.org/10.1016/j.scitotenv.2014.01.061 
Bodoque, J., Amérigo, M., Díez-Herrero, A. (2016). Improvement of resilience of urban areas by integrating social perception in flash-flood risk management, J. Hydrol., 541, p. 665-676.

DOI: http://dx.doi.org/10.1016/j.jhydrol.2016.02.005

Câmara, A., Ferreira, C., Silva, L., Alves, M. e Brazão, M. (2001). Geografia. Orientações Curriculares $3^{\circ}$ Ciclo. Dep. de Educação Básica. Ministério da Educação.

CONSELHO NACIONAL DE EDUCAÇÃO - EDUCAÇÃO PARA O RISCO, Recomendação n. ${ }^{\circ}$ 5/2011, Ministério da Educação e Ciência, Diário da República, $2{ }^{\mathrm{a}}{ }^{\text {série }}-\mathrm{N} .{ }^{\circ} 202-20$ de Outubro de 2011.

Cunha, L. e Cravidão, F. (2001). Territorio, urbanización y calidad medioambiental: una trilogía incompatible?, Actas del II Encuentro Internacional de Estudios Urbanos, La Habana.

Cunha, L. e Dimuccio, L. (2002). Considerações sobre riscos naturais num espaço de transição. Exercícios cartográficos numa área a Sul de Coimbra, Territorium n. ${ }^{a}$ 9, Coimbra, p. 37-53. Disponível em: http://www.uc.pt/fluc/nicif/riscos/Documentacao/ Territorium/T09_artg/T09_artg03.pdf

Dixit (2003). Floods and vulnerability: need to rethink flood management. In: Natural Hazards. vol. 28, pp. 155-179.

Faugères, L. (1990). La dimension des faits et la théorie du risque. Le Risque et la Crise, European Coordination Centre for Research and Documentation in Social Sciences, Foundation for International Studies, Malta, p. 31-60.

Granger-Morgan (1997). Public perception, understanding, and values, in: The industrial green game: Implications for environmental design and management, edited by: Richards, D. J., National Academy Press, Washington DC, 200-211.

Kaufman, L. R. P. J. (1990). Finding Groups in Data: An Introduction to Cluster Analysis. 9 edition.

Lebel (2006). Invisible stripes? Formerly incarcerated persons' perceptions of and responses to stigma. Dissertation Abstracts International, 67 (2-A), 731A. (UMI No. 0419-4209).

Martin, P. (1998). Ces risques que l'on dit naturels, EDISUD, 256 p.

McGee e Russel (2003). It's just a natural way of life...: An investigation of wildfire preparedness in rural Australia. Environmental Hazards, 5(1), p. 1-12. DOI: http://dx.doi.org/10.1016/j.hazards.2003.04.001

Miceli, R., Sotgiu, E., Settanni, M. (2008). Disaster Preparedness and Perception of Flood Risk: A study in an Alpine Valley in Italy. Journal of Environmental Psychology, 28, p.164-173.

DOI: http://dx.doi.org/10.1016/j.jenvp.2007.10.006
Nir, D. (1983). Man, a geomorphological agent. An introduction to Antropic Geomorphology, Jerusalem, Keter Publishing House, 165 p.

NRC - NATIONAL RESEARCH COUNCIL, COMMITTEE ON GEOGRAPHY (2006). Learning to Think Spatially: GIS as a Support System in the K-12 Curriculum (1 $1^{\text {st }}$ ed.). National Academies Press.

Nunes, A., Almeida, A., C. e Nolasco, C. (2013). Educação para o Risco: contributo da Geografia no $3 .^{\circ}$ ciclo do Ensino Básico. In L. Lourenço e M. Mateus (Coord. e Org.), Riscos naturais, antrópicos e mistos. Homenagem ao Professor Doutor Fernando Rebelo. Departamento de Geografia. Faculdade de Letras. Universidade de Coimbra, p. 123-132. Disponível em: https://www.uc.pt/fluc/depgeo/Publicacoes/livro_ homenagem_FRebelo/143_152

Nunes, A., Oliveira, S., Lourenço, L., Vieira, A., BentoGonçalves, A. e Félix, F. (2015). Vulnerabilidade a incêndios na Europa Mediterrânea. Abordagem conceptual e a utilização de dados de satélite. In J. G. Dos Santos, C. Fonte, R. F. de Figueiredo, A. Cardoso, G. Gonçalves, J. P. Almeida w A. Baptista (Eds), Atas das I Jornadas Lusófonas Ciências e Tecnologias de Informação Geográfica, Marcos e Marcas Lusófonas. Coimbra, 11 a 13 de Setembro de 2014, p. 330-344. DOI: http://dx.doi.org/10.14195/978-989-26-0983-6_18

Oliveira, S., Laneve, G., Fusilli, L., Eftychidis, G., Nunes, A., Lourenço, L. and Sebastián-Lopéz, A. (2017). A Common Approach to Foster Prevention and Recovery of Forest Fires in Mediterranean Europe. In Borna Fuerst-Bjeliš (Ed.), "Mediterranean Identities - Environment, Society, Culture”. Chapter 14, InTech- Open Access Publisher, pp. 337-361.

Panizza, M. (1990). Geomorfologia applicata al rischio i al impatto ambientali. Un esempio nelle dolomiti (Italia), Teruel, Actas de la $1^{a}$ Reunión Nacional de Geomorfología, Vol.1, p.1-16.

Pestana, M. H. e Gageiro, J. N. (1998) Análise de dados para Ciências Sociais-A complementaridade do SPSS. Edições Sílabo, Lisboa.

Plapp e Werner (2006). Understanding risk perception from natural hazards: examples from Germany. In: RISK 21 - Coping with risks due to natural hazards in the $21^{\text {st }}$ century. W. Ammann, S. Dannenmann, L. Vulliet (eds.). Rotterdam: Taylor and Francis/ Balkema, London, p. 101-108.

Ranger-Morgan (1997). Public perception, understanding, and values, in: The industrial green game: Implications for environmental design and management, edited by: Richards, D. J., National Academy Press, Washington DC, p. 200-211. 
Rebelo, F. (1995). Os conceitos de risco, perigo e crise e a sua aplicação ao estudo dos grandes incêndios florestais. Biblos, 71, p. 511-527.

Rebelo, F. (2008). Um novo olhar sobre os riscos? OS exemplos das cheias rápidas (flash floods) em domínio mediterrâneo, Territorium, n¹5, Coimbra, p. 7-14. Disponível em: http://www.uc.pt/fluc/nicif/riscos/ Documentacao/Territorium/T15_artg/T15art02.pdf

Silva, L., Ferreira, C. (2000). O cidadão geograficamente competente: competências da geografia no ensino básico. Inforgeo, 15, Lisboa, Edições Colibri, p. 91-102.

Slovic, P. (1987). Perception of Risk. Science, Vol. 236, p. $280-285$.
Smith, K. (1996). Environmental hazards. Assessing risks and reducing disasters, London, Routledge, 389 p.

Tedim, F., Ferreira, M., Cunha, M. A. e Sousa, C. G. (2010). Risco de Incêndio Florestal no Ensino da Geografia no $3^{\circ}$ Ciclo do Ensino Básico. Ensino da Geografia e Processo de Bolonha. Actas do XII Colóquio Ibérico de Geografia, 6 a 9 de Outubro Porto: Faculdade de Letras, Universidade do Porto.

Terpstra, T. (2009). Flood preparedness: thoughts, feelings and intentions of the Dutch public. Thesis, University of Twente. 